\title{
Oxiracetam ameliorates cognitive deficits in vascular dementia rats by regulating the expression of neuronal apoptosis/autophagy-related genes associated with the activation of the Akt/mTOR signaling pathway
}

\author{
Jing Xu(i), Qianqian Qi(i), Peiyuan Lv(i), Yanhong Dong (ib), Xin Jiang(i), and Zhijuan Liu(i) \\ Department of Neurology, Hebei General Hospital, Shijiazhuang City, Hebei Province, China
}

\begin{abstract}
Oxiracetam (ORC) is a commonly used nootropic drug for improving cognition and memory impairments. The therapeutic effect and underlying mechanism of ORC in vascular dementia $(\mathrm{VaD})$ treatment remain unknown. In this study, 3-month-old male Sprague-Dawley rats with permanent bilateral common carotid artery occlusion-induced $\mathrm{VaD}$ were treated orally with low $(100 \mathrm{mg} / \mathrm{kg})$ or high $(200 \mathrm{mg} / \mathrm{kg})$ dose ORC once a day for 4 weeks. The results of the Morris water maze test and Nissl staining showed that ORC treatment significantly alleviated learning and memory deficits and neuronal damage in rats with VaD. Mechanistically, the protein levels of a panel of genes associated with neuronal apoptosis (Bcl-2, Bax) and autophagy (microtubule-associated protein 1 chain 3 , Beclin1, p62) were significantly altered by ORC treatment compared with VaD, suggesting a protective role of ORC against VaD-induced neuronal apoptosis and autophagy. Moreover, the Akt/mTOR pathway, which is known to be the upstream signaling governing apoptosis and autophagy, was found to be activated in ORCtreated rats, suggesting an involvement of Akt/mTOR activation in ORC-rendered protection in VaD rats. Taken together, this study demonstrated that ORC may alleviate learning and memory impairments and neuronal damage in VaD rats by altering the expression of apoptosis/autophagy-related genes and activation of the Akt/mTOR signaling pathway in neurons.
\end{abstract}

Key words: Oxiracetam; Vascular dementia; Bilateral common carotid artery occlusion; Autophagy; Apoptosis

\section{Introduction}

Vascular dementia $(\mathrm{VaD})$ is the most common cause of dementia in the elderly, second to Alzheimer's disease (AD), and is characterized by progressive cognitive and behavioral deterioration resulting from vascular events that damage blood vessels and impair blood flow in the brain $(1,2)$. Neural lesions and neurodegeneration in various brain regions accompanied by learning and memory deficits have been implicated in $\mathrm{VaD}(2)$. Despite recent developments in experimental and clinical neuroscience, no drug has been approved to date for treatment of $\mathrm{VaD}$ in the world (3). It is urgently required to find an effective pharmacological compound against $\mathrm{VaD}$ due to its high prevalence and morbidity, and lack of effective treatment options.

It is well-established that the balance of protein expression between antiapoptotic $\mathrm{Bcl}-2$ and proapoptotic Bax plays an important role in cell apoptosis (4). A recent study demonstrated that decreased $\mathrm{Bcl}-2 / \mathrm{Bax}$ ratio contributes to neuron apoptosis in the hippocampus of rats with permanent bilateral common carotid artery occlusion (BCCAO), a widely used model of $\operatorname{VaD}(5,6)$. In addition, activation of the PI3K/Akt pathway can protect neurons from apoptosis in cerebral ischemia-reperfusion (IR) injury via the regulation of $\mathrm{Bax}$ and $\mathrm{Bcl}-2$ expression $(7,8)$. These data suggest Bcl-2/Bax-modulated apoptosis as a potential therapeutic target for prevention and intervention of BCCAO-induced VaD. Furthermore, accumulating evidence indicates that autophagy is another form of programmed neuronal cell death that is triggered by intracellular buildup of pathogenic proteins and damaged organelles $(9,10)$. Modulation of autophagy may serve as an alternative therapeutic strategy in brain disorders such as ischemic stroke, AD, Parkinson's disease, and Huntington's disease (11). Intriguingly, the interplay between apoptosis and autophagy may cause coexistence of both

Correspondence: Peiyuan Lv: <peiyuanlu2@163.com>

Received January 30, 2019 | Accepted September 4, 2019 
of them in the same cell (12). Mammalian target of rapamycin (mTOR), one of the target genes of the PI3K/Akt pathway, which directly regulates the autophagy cascade, appears to bridge the interaction between apoptosis and autophagy $(13,14)$. Several previous studies have provided some evidence that the activation of $A k t / m T O R$ signaling is involved in the protective effect against vascular cognitive impairment $(8,15-18)$. Various models of IR injury treated with various molecules revealed that IR injury is mitigated through Akt/mTOR signaling (19-24), promoting cell survival.

Oxiracetam (ORC; 4-hydroxy-2-oxo-1-pyrrolidine acetamide), a derivative of $\gamma$-aminobutyric acid, is a commonly used nootropic drug for improving cognition and memory impairments (25). ORC can diffuse across the blood-brain barrier and extensively distribute in the hippocampus, cerebral cortex, and striatum with high concentration (2628 ), remarkably improving the clinical outcomes of various brain disorders, including neurodegenerative diseases, cognition and memory deficits, seizure, stroke, and anxiety (29). ORC is one of the most common nootropic drugs used for the management of cerebrovascular impairments, including $\mathrm{VaD}$, and has shown beneficial effects $(25,30,31)$. Yao et al. (32) showed that ORC has beneficial effects in rats with cognitive impairment due to chronic cerebral hypoperfusion. Previous studies demonstrated that the beneficial role of ORC in brain disorders is mainly attributable to its abilities to promote the synthesis of lipids, proteins, and nucleic acids as well as increase the levels of high-energy phosphates and membrane-bound protein kinase $C$ in neurons, which leads to an enhancement of metabolism in the brain $(33,34)$. The mechanism of action of ORC is also hypothesized to involve energy metabolism in the brain (33). Nevertheless, whether ORC influences neuronal apoptosis and autophagy is unknown. In addition, whether ORC has a protective role in BCCAO-induced $\mathrm{VaD}$ and the underlying mechanisms are poorly understood.

In this study, we established a rat model of BCCAOinduced $\mathrm{VaD}$ treated with low- or high-dose ORC to explore the therapeutic effect of ORC on cognition impairments and neuron damage using the Morris water maze (MWM) test and Nissl staining, respectively. To better understand the molecular mechanism underlying ORC-reduced cognitive decline and neuron loss, the expression of a panel of proteins associated with apoptosis and autophagy was determined. The results demonstrated that ORC could improve cognition impairment and brain damage in $\mathrm{VaD}$ rats through modulation of apoptosis and autophagy in neuronal cells.

\section{Material and Methods}

\section{Animals and surgery}

Forty Sprague-Dawley male rats (3 months old, 250$300 \mathrm{~g}$ ) were obtained from the Laboratory Animal Center of Hebei Medical University, China, and maintained in an environmentally controlled room at $23 \pm 1^{\circ} \mathrm{C}$ with a 12-h light/dark cycle and free access to water and food. All animal procedures were approved by the Animal Care and Use Committee of Hebei General Hospital (China) (approval \#201903) and carried out in accordance with the Regulations of Laboratory Animal Management issued by the Ministry of Science and Technology of the People's Republic of China.

The rats were randomly divided into four groups $(n=10 /$ group): sham group, $\mathrm{VaD}+$ saline group ( $\mathrm{VaD}), \mathrm{VaD}+$ low-dose ORC group (ORC-L), and $\mathrm{VaD}+$ high-dose ORC group (ORC-H). Rats were anesthetized intraperitoneally (ip) with pentobarbital sodium (50 mg/kg), and a 28-gauge single guide cannula was implanted into the left lateral ventricle of each rat using the following coordinates: $0.8 \mathrm{~mm}$ posterior to bregma, $1.5 \mathrm{~mm}$ lateral to the midline, and $3.8 \mathrm{~mm}$ below the skull surface. The rats were allowed to recover for 7 days before BCCAO to induce $\mathrm{VaD}$ as previously described (35). Briefly, rats were anesthetized with pentobarbital sodium (50 mg/kg, ip). The bilateral common carotid arteries were exposed and gently separated from the carotid sheath and vagus nerve through a ventral cervical incision followed by doubleligation with 4-0 silk sutures and cutting between the ligations. Sham rats underwent the same surgical procedures without occlusion of the carotid arteries. During the surgical procedure, the body temperature was maintained at $36.5-37.5^{\circ} \mathrm{C}$ using a heat lamp. After surgery, rats were kept in the animal resource facility with free access to food and water.

\section{ORC administration}

ORC powder $(99.6 \%$ purity) was obtained from Shijiazhuang Pharmaceutical Group NBP Pharmaceutical Co., Ltd. (China) and diluted to $50 \mathrm{mg} / \mathrm{mL}$ in physiological saline. One day after BCCAO surgery, the ORC-L and ORC-H groups were orally treated with ORC at 100 and $200 \mathrm{mg} / \mathrm{kg}$, respectively, once a day for 4 weeks, while the sham and saline groups were treated with an equal volume of saline.

\section{MWM training and test}

A circular pool $(120 \mathrm{~cm}$ in diameter, $60 \mathrm{~cm}$ in height; Shanghai Jiliang Software Technology, China), with a video camera hanging over it, was filled to a depth of $45 \mathrm{~cm}$ with water at $23 \pm 1^{\circ} \mathrm{C}$ and was located in a dimly lit, quiet test room. Extramaze visual cues were at fixed positions and could be used for spatial orientation by the rats. The maze was divided into four equal quadrants (Northeast, Southeast, Southwest, and Northwest) by placing four poles at the four cardinal points (East, West, North, and South) along the perimeter of the pool. A transparent escape platform was submerged approximately $1 \mathrm{~cm}$ below the surface of the water and located in the center of one quadrant during training. From day 1 following drug treatment, each rat received six training 
trials per day for 5 consecutive days. During each trial, the rats were gently placed in the water and faced toward the wall of the maze from four cardinal points. The time spent to find the hidden platform (escape latency) and the swimming trajectories were recorded. If a rat failed to find the platform within $60 \mathrm{~s}$, the rat would be guided to the hidden platform by the researcher and was allowed to stay on the platform for $15 \mathrm{~s}$ before being removed from the water, and a maximum score of $60 \mathrm{~s}$ was assigned. Animals with swim speeds less than $10 \mathrm{~cm} / \mathrm{s}$ were excluded from further analysis due to low motivation and failure to perform the task. On day 6 , the hidden platform was removed and the rats underwent a probe test for $60 \mathrm{~s}$. The percentage of time spent in the target quadrant where the platform was located was recorded.

\section{Nissl staining \\ At the end of drug treatment, all rats were anesthetized with $1 \%$ pentobarbital sodium $(50 \mathrm{mg} / \mathrm{kg}$, ip) and per- fused with $0.9 \%$ saline followed by $4 \%$ paraformaldehyde through the left cardiac ventricle and ascending aorta. The brain of each rat was immediately removed. Four brain samples were randomly selected from each group, fixed in $4 \%$ paraformaldehyde for $48 \mathrm{~h}$, and embedded in paraffin. The hippocampal CA1 area was cut into $5-\mu \mathrm{m}$ thick sections, and Nissl staining was performed with $1 \%$ toluidine blue according to the manufacturer's instructions. Two slides were randomly selected from the same site of each rat and were observed under a 50i light microscope (Nikon, Japan). Images were acquired at $\times 400$ magnification using a Nikon camera (Japan).}

\section{Western blot assay}

The hippocampal tissues of six rats randomly selected from each group were removed from the brains and the proteins were extracted using ice-cold radioimmunoprecipitation assay (RIPA) buffer, according to the manufacturer's instructions (Solarbio, China), followed by an incubation on ice for $30 \mathrm{~min}$. The homogenates were centrifuged at $120,000 \mathrm{~g}$ for $15 \mathrm{~min}$ at $4^{\circ} \mathrm{C}$, and the supernatants were collected. Protein concentration was measured using the bicinchoninic acid method (Pierce, USA). Protein samples were separated by $12 \%$ sodium dodecyl sulfate-polyacrylamide gel electrophoresis and transferred onto polyvinylidene fluoride (PVDF) membranes. After blocking with 5\% fat-free milk in Tris-buffered saline and Tween-20 (TBST) for $2 \mathrm{~h}$, the membranes were incubated with primary rabbit antibodies for Akt (1:1000; Epitomics, USA), p-Akt Ser473 (1:1000; Epitomics), Bcl-2 (1:1000; Cell Signaling Technology, USA), Bax (1:1000; Cell Signaling Technology), mTOR (1:500; Epitomics), pmTOR Ser2448 (1:500; Epitomics), LC3B (1:500; Abgent, USA), p62 (1:2000; Abcam, USA), or $\beta$-actin (1:5000, 1:2000; Santa Cruz, USA) overnight at $4^{\circ} \mathrm{C}$. $\beta$-actin was used as an internal control. Following three washes with TBST, the membranes were incubated with horseradish peroxidase-conjugated secondary antibodies (1:1000, goat anti-rabbit $\mathrm{lgG}$ ) for $1 \mathrm{~h}$ at room temperature. The protein bands on the membranes were detected with the enhanced chemiluminescent reagent (Solarbio). The densitometry values were determined using ImageJ (version 1.3; NIH, Wayne Rasband, USA) and normalized to $\beta$-actin.

\section{Statistical analysis}

All data are reported as means $\pm S E$. Statistical analysis was performed using SPSS 16.0 (IBM, USA). Differences in the escape latencies in the MWM test were analyzed using the two-way analysis of variance (ANOVA). Statistical significance among multiple groups was assessed using the Student-Newman-Keuls (SNK) test. Other comparisons were conducted using one-way ANOVA followed by the SNK test. $\mathrm{P}<0.05$ was considered statistically significant.

\section{Results}

ORC ameliorated learning and memory impairments

The results of the MWM test showed that the swimming trajectory length and escape latency in the $\mathrm{VaD}$ group were significantly increased compared with those in the sham group, which indicated learning impairment in rats with BCCAO-induced VaD. Compared with the $\mathrm{VaD}$ group, ORC treatment significantly decreased swimming trajectory length and escape latency as early as 3 days post treatment in both ORC-treated groups in a dose-dependent manner $(F=51.132$ for intergroup comparison; $\mathrm{P}<0.05$ ) (Figure $1 \mathrm{~A}$ and $\mathrm{B}$ ), suggesting that ORC could improve the learning ability of rats with $\mathrm{VaD}$. Furthermore, in the probe test, rats in the $\mathrm{VaD}$ group spent significantly less time in the target quadrant than those in the sham group $(F=15.009 ; P<0.01)$, and rats in the ORC-L and ORC-H groups spent more time than those in the $\mathrm{VaD}$ group $(\mathrm{F}=15.009 ; \mathrm{P}<0.01)$ (Figure $1 \mathrm{C}$ and $\mathrm{D}$ ), suggesting a memory-improving effect of ORC in $\mathrm{VaD}$ rats. Taken together, these data demonstrated that ORC may be an effective therapeutic agent in reducing learning and memory deficits in rats with $\mathrm{VaD}$.

\section{ORC reversed neuropathological alterations}

In the sham group, neuron cells with normal size and morphology, clear nucleus, and Nissl substance in the cytoplasm were tightly packed and orderly arranged. In contrast, neurons with loss of Nissl substance and abnormal nuclear shape were loosely and irregularly arranged in the $\mathrm{VaD}$ group. In addition, there was a significant loss of neurons observed in the saline group compared with the sham group (Figure 2). These neuropathological changes and neuron loss were significantly improved in ORC-treated mice, suggesting a protective role of ORC against neuronal damage in $\mathrm{VaD}$. 
A

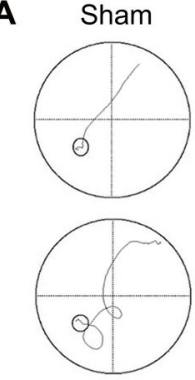

ORC-L

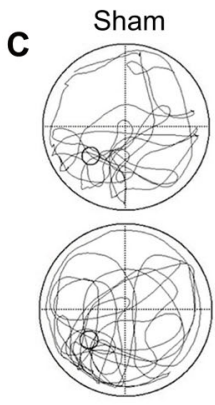

ORC-L

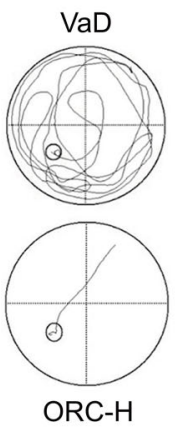

$\mathrm{VaD}$

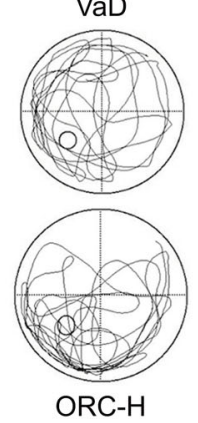

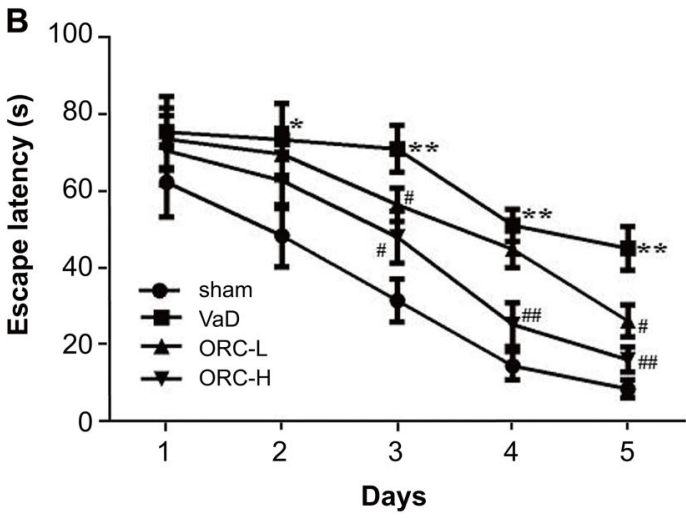

$\mathrm{D}$

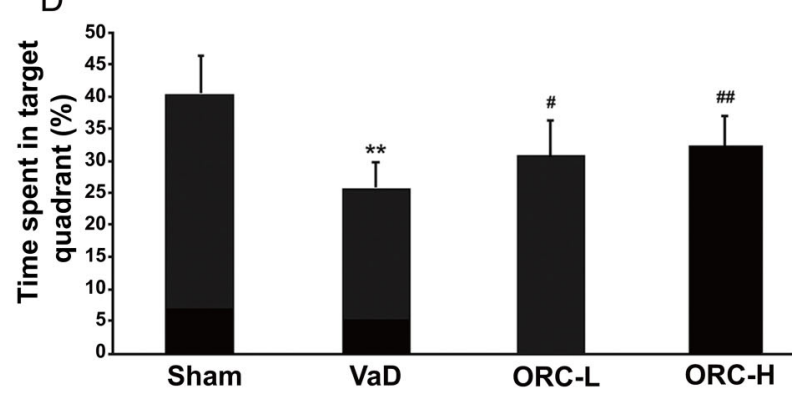

Figure 1. Effects of oxiracetam (ORC) on spatial learning and memory impairments in rats with permanent bilateral common carotid artery occlusion (BCCAO)-induced vascular dementia $(\mathrm{VaD})$ assessed by the Morris water maze test. The swimming trajectories $(\mathbf{A})$ and the time spent to find the hidden platform (escape latency; B) were recorded daily during training to assess the learning ability. A probe test was performed on day 6 post-ORC treatment to test the memory of the rats. The swimming trajectories (C) and the time spent in the target quadrant where the platform was located (D) were recorded. ${ }^{*} \mathrm{P}<0.05$, ${ }^{* *} \mathrm{P}<0.01$ vs sham group; ${ }^{\#} \mathrm{P}<0.05$, ${ }^{\# \#} \mathrm{P}<0.01$ vs saline (VaD) group; $n=10$ for each group (ANOVA). ORC-L: low-dose oxiracetam; ORC-H: high-dose oxiracetam.

ORC altered the expression of apoptosis/autophagyrelated genes in the hippocampus of rats

As shown in Figure $3 \mathrm{~A}$ and $\mathrm{B}$, the decreased $\mathrm{Bcl}-2 / \mathrm{Bax}$ ratio in the $\mathrm{VaD}$ group was significantly restored by ORC treatment regardless of dose, suggesting an inhibitory role of ORC in neuronal apoptosis. In addition, the conversion of microtubule-associated protein 1 chain 3 (LC3) to phosphatidylethanolamine-conjugated LC3 (LC3-II), which reflects the number of autophagosomes or the degree of autophagy (36), was significantly decreased in the ORC-H group $(F=15.577 ; P<0.05)$, but not in the ORC-L group, compared with that in the $\mathrm{VaD}$ group (Figure $3 \mathrm{C}$ ), suggesting a protective role of ORC against autophagy at the high dose. Similar trends were also observed in the expression of other autophagy-related genes, such as Beclin1 and p62 (Figure 3D and E). Taken together, these data indicated that ORC may protect neuron cells from apoptotic and autophagic cell death through altering the expression of a variety of related genes.

ORC induced phosphorylation of Akt and mTOR in the hippocampus of rats

Considering that Akt signaling is involved in neuronal apoptosis and that mTOR bridges the interplay between apoptosis and autophagy $(13,14)$, we next sought to investigate whether ORC could change the levels of activated Akt and mTOR to reveal the signaling upstream ORC-suppressed apoptosis/autophagy. As shown in Figure 4 A-C, both phosphorylated Akt (p-Akt) and phosphorylated mTOR ( $p$-mTOR) were significantly increased in ORC-treated groups (p-Akt: $F=25.301$; p-mTOR: $\mathrm{F}=21.183$; both $\mathrm{P}<0.05$ ) compared with the $\mathrm{VaD}$ group in a dose-dependent manner, while the protein levels of total Akt and total mTOR remained unchanged. These data suggested that the protective role of ORC against BCCAO-induced neuronal apoptosis/autophagy was mediated by the activation of the Akt/mTOR signaling pathway.

\section{Discussion}

$\mathrm{VaD}$ is characterized by progressive cognitive impairment and extensive neuropathological lesions in brain tissues due to chronic cerebral hypoperfusion (1-3,37). An effective therapeutic approach is urgently required for reducing cognitive degeneration and neuronal damages in $\mathrm{VaD}$. In this study, the results of the MWM test showed remarkable poorer learning and memory skills of rats in 
Sham

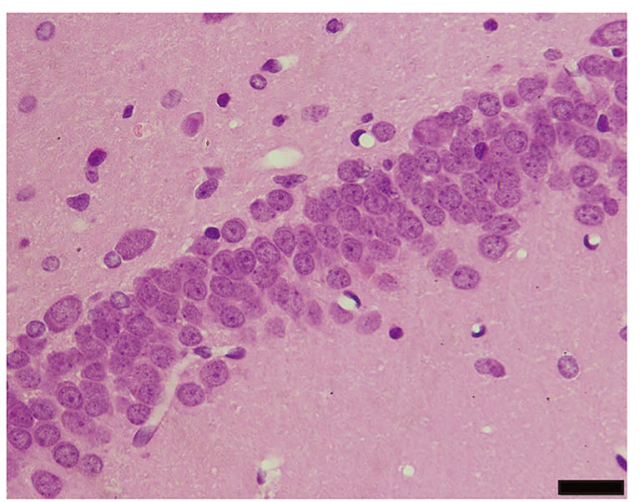

ORC-L

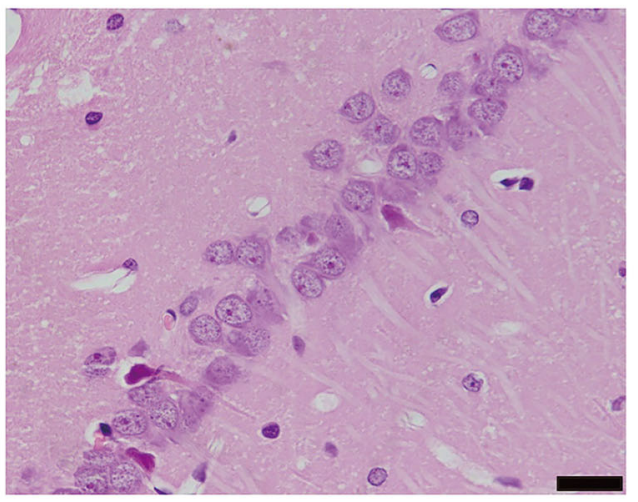

$\operatorname{VaD}$

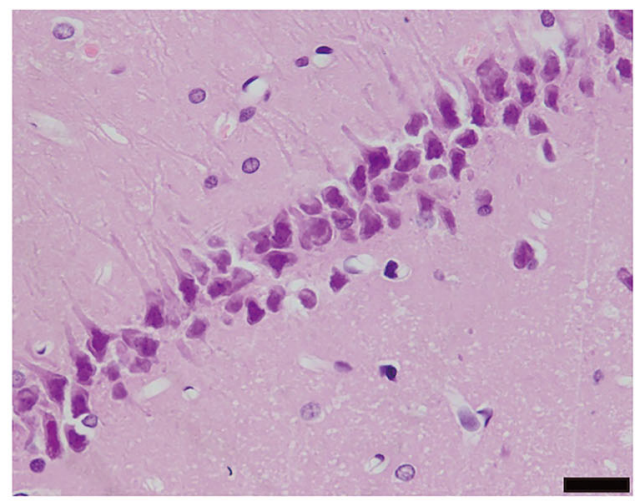

ORC-H

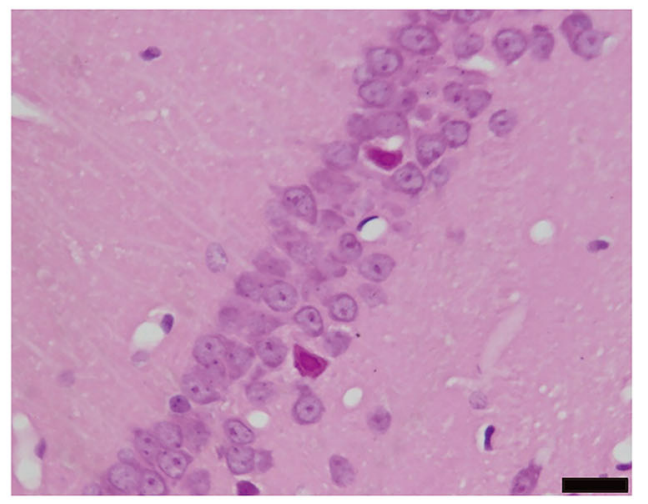

Figure 2. Nissl staining in the hippocampus CA1 region of rats. Magnification 400x; scale bar: $20 \mu \mathrm{m}$. ORC-L: low-dose oxiracetam; ORC-H: high-dose oxiracetam; VaD: vascular dementia. In the sham group, neuron cells with normal size and morphology, clear nucleus, and Nissl substance in the cytoplasm were tightly packed and orderly arranged. In the VaD group, obvious pathologic changes were evident, including loosely arranged neurons, neuronal shrinkage and loss. These neuropathological changes and neuron loss were significantly improved in ORC-treated mice, and the improvement in the ORC-H group was more obvious than ORC-L group.

the saline group than those in the sham group. A consecutive daily administration of ORC at 100 or $200 \mathrm{mg} / \mathrm{kg}$ dosedependently improved the impaired learning and memory abilities of $\mathrm{VaD}$ rats as evidenced by markedly decreased escape latencies in the training sessions and increased time spent in the target quadrant in the probe trial compared with the saline group. Similar trends were also observed in Nissl staining for neurons. These results are consistent with previous research (38-40), suggesting that the rat model of $\mathrm{VaD}$ used in the present study is reliable.

To explain why ORC treatment can reduce neuron damage and loss in the hippocampus of $\mathrm{VaD}$ rats, as shown by Nissl staining, we explored the expression of a variety of genes associated with apoptosis and autophagy, as they are two major processes involved in neuronal damages and death $(5,6,9,10)$. Several previous studies have provided some evidence that the activation of Akt/ mTOR signaling is involved in the protective effect against vascular cognitive impairment (8,15-18). Compared with the sham group, the $\mathrm{VaD}$ groups showed some baseline activation of Akt/mTOR signaling, which is probably a response to the IR injuries. Consistent with previous studies showing that the imbalance between antiapoptotic $\mathrm{Bcl}-2$ and apoptotic Bax in repeated cerebral IR and chronic cerebral hypoperfusion are two common events in $\mathrm{VaD}$ animal model $(5,41,42)$, we found that the ratio of Bcl2/Bax was significantly decreased in $\mathrm{VaD}$ rats compared with the sham ones, suggesting an enhancement of neuronal apoptosis in $\mathrm{VaD}$ rats. Interestingly, the decreased ratio of $\mathrm{Bcl}-2 / \mathrm{Bax}$ in $\mathrm{VaD}$ rats was significantly restored upon treatment with ORC regardless of dose, suggesting a neuroprotective role of ORC against apoptotic cell death. The present study suggests that oxiracetam treatment could further increase AKT/mTOR signaling to protect the cells against apoptosis and autophagy, as shown in various models of IR injury and various molecules used to mitigate its damage (19-24), and hence 

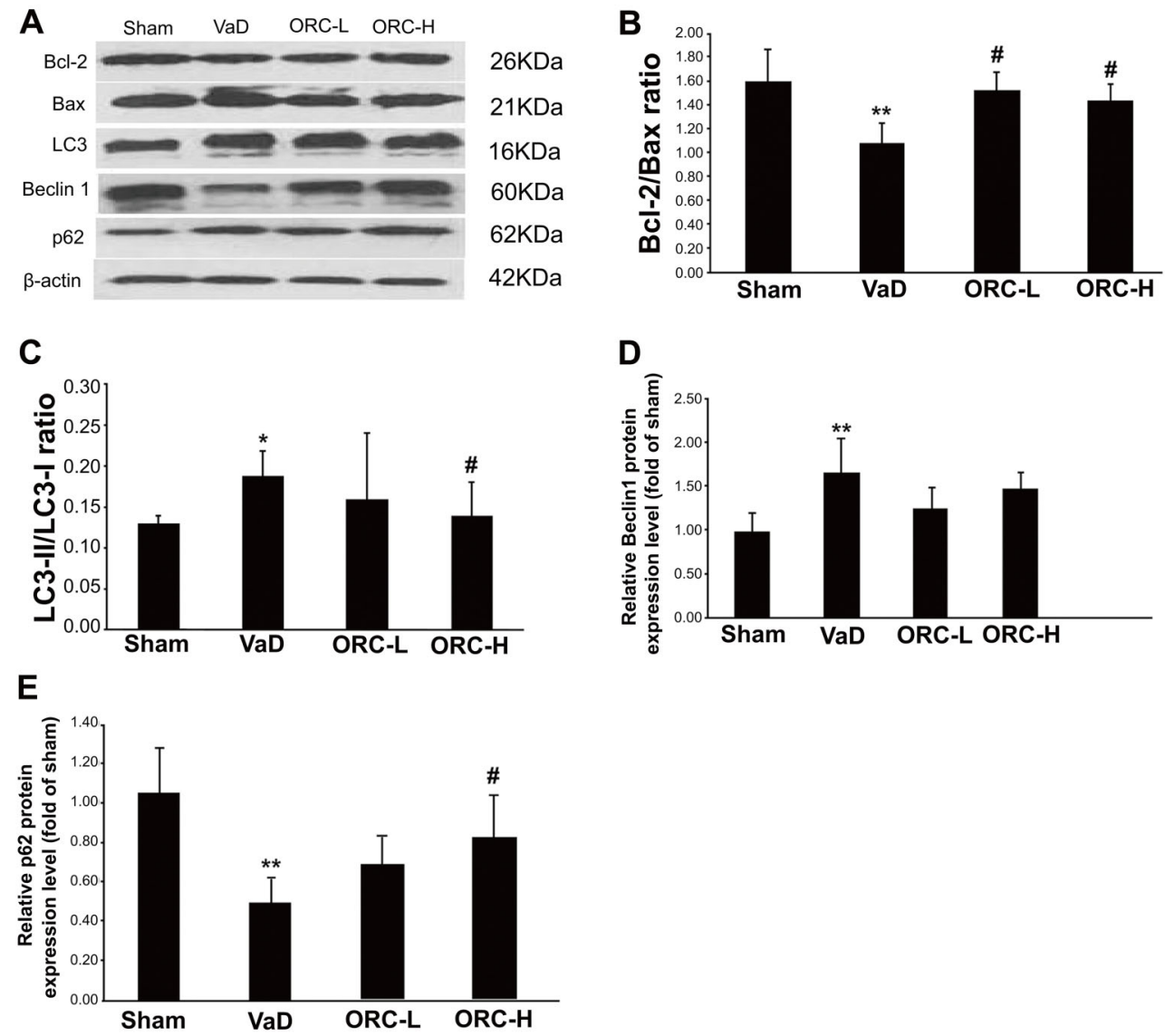

Figure 3. Alteration of apoptosis- and autophagy-related gene expression in response to oxiracetam (ORC). A, The protein levels of $\mathrm{Bcl}-2$, Bax, LC3, Beclin1, and p62 in the hippocampus of rats were determined by western blot analysis after 4 weeks of ORC treatment. B, Bcl-2/Bax ratio. C, LC3-II/LC3-I ratio. D and E, Quantification of Beclin1 and p62 protein expression. $\beta$-actin was used as the internal control. ${ }^{*} \mathrm{P}<0.05$, ${ }^{* *} \mathrm{P}<0.01$ vs sham group; ${ }^{\#} \mathrm{P}<0.05$, ${ }^{\# \#} \mathrm{P}<0.01$ vs $\mathrm{VaD}$ group; $\mathrm{n}=10$ for each group (ANOVA). VaD: vascular dementia; ORC-L: low-dose oxiracetam; ORC-H: high-dose oxiracetam; LC3: microtubule-associated protein 1 chain 3; LC3-II: phosphatidylethanolamine-conjugated LC3.

promoting cell survival. Moreover, dysregulation of autophagy markers, including LC3, Beclin1, and p62, was observed in the hippocampus of $\mathrm{VaD}$ rats compared with sham ones, suggesting an involvement of autophagy in VaD-induced neuron damage and loss. Although autophagy is primarily a protective process for cell survival, it seems that autophagy has a dual role in the central nervous system (43). Excessive autophagy exerts a detrimental effect in neurological disease, leading to autophagic neuronal death (10). ORC-induced reversal of dysregulated expression of LC3, Beclin1, and p62 indicates that targeting autophagy is another possible mechanism underlying the protective role of ORC in VaD. However, the exact role of autophagy in $\mathrm{VaD}$ needs further investigation.

To further clarify the signaling pathway upstream ORCregulated apoptosis/autophagy, the phosphorylation status of $A k t / m T O R$ was assessed because $A k t / m T O R$ signaling is involved in the interplay between apoptosis and autophagy $(13,14)$. It has been reported that phosphorylation of Akt can suppress IR-induced apoptotic cell death in neurons through upregulation of antiapoptotic genes and downregulation of proapoptotic ones (44). Our results showed that ORC treatment significantly upregulated $p$-Akt and $p$-mTOR expression in VaD rats, suggesting an involvement of Akt/mTOR activation in ORC-rendered protection against VaD-induced apoptosis/ autophagy.

In conclusion, the present work demonstrated that ORC could attenuate cognitive impairment and neuronal damages in BCCAO-induced $\mathrm{VaD}$ in rats possibly through regulation of neuronal apoptosis- and autophagy-related genes via activation of the Akt/mTOR signaling pathway, which provides ORC as an effective therapeutic compound targeting neuronal apoptosis/autophagy in $\mathrm{VaD}$ treatment. Further studies are required to reveal the precise effect and mechanism of ORC in VaD therapy. 

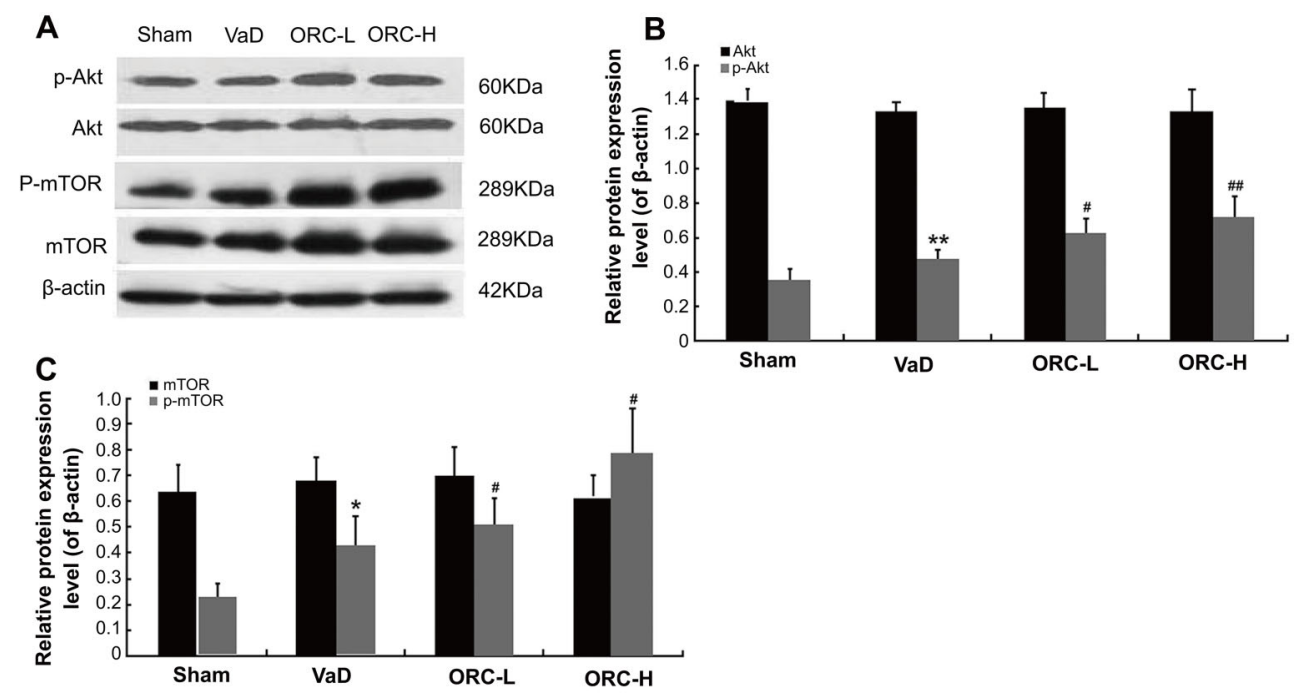

Figure 4. Alteration of apoptosis- and autophagy-related gene expression in response to oxiracetam (ORC). A, The protein levels of total Akt, phosphorylated Akt (p-Akt), total mTOR and phosphorylated mTOR (p-mTOR) in the hippocampus of rats were determined by western blot analysis after 4 weeks of ORC treatment. B and C, Quantification of panel A. $\beta$-actin was used as an internal control. ${ }^{*} \mathrm{P}<0.05$, ${ }^{* *} \mathrm{P}<0.01$ vs sham group; ${ }^{\#} \mathrm{P}<0.05$, ${ }^{\# \#} \mathrm{P}<0.01$ vs saline $(\mathrm{VaD})$ group; $\mathrm{n}=10$ for each group (ANOVA). VaD: vascular dementia; ORC-L: low-dose ORC; ORC-H: high-dose ORC; mTOR: mammalian target of rapamycin.

\section{Acknowledgments}

This study was supported by The National Natural Science Foundation of China (Grant No. 81241037), the

\section{References}

1. Tarawneh R, Holtzman DM. The clinical problem of symptomatic Alzheimer disease and mild cognitive impairment. Cold Spring Harb Perspect Med 2012; 2: a006148, doi: 10.1101/cshperspect.a006148.

2. Khan A, Kalaria RN, Corbett A, Ballard C. Update on vascular dementia. J Geriatr Psychiatry Neurol 2016; 29: 281-301, doi: 10.1177/0891988716654987.

3. Baskys A, Cheng JX. Pharmacological prevention and treatment of vascular dementia: approaches and perspectives. Exp Gerontol 2012; 47: 887-891, doi: 10.1016/j.exger. 2012.07.002.

4. Sun B, Chen L, Wei X, Xiang Y, Liu X, Zhang X. The Akt/ GSK-3beta pathway mediates flurbiprofen-induced neuroprotection against focal cerebral ischemia/reperfusion injury in rats. Biochem Biophys Res Commun 2011; 409: 808-813, doi: 10.1016/j.bbrc.2011.05.095.

5. Jin $\mathrm{W}$, Jia $\mathrm{Y}$, Huang $\mathrm{L}$, Wang $\mathrm{T}$, Wang $\mathrm{H}$, Dong $\mathrm{Y}$, et al. Lipoxin A4 methyl ester ameliorates cognitive deficits induced by chronic cerebral hypoperfusion through activating ERK/Nrf2 signaling pathway in rats. Pharmacol Biochem Behav 2014; 124: 145-152, doi: 10.1016/j.pbb.2014.05.023.

6. Jing Z, Shi C, Zhu L, Xiang Y, Chen P, Xiong Z, et al. Chronic cerebral hypoperfusion induces vascular plasticity and hemodynamics but also neuronal degeneration and cognitive impairment. J Cereb Blood Flow Metab 2015; 35: 12491259, doi: 10.1038/jcbfm.2015.55.
Hebei Province Major Medical Research Project of China (zd2013005) and the Hebei Province Natural Science Foundation of China (H2013307046).

7. Ma Y, Lu C, Li C, Li R, Zhang Y, Ma H, et al. Overexpression of HSPA12B protects against cerebral ischemia/reperfusion injury via a PI3K/Akt-dependent mechanism. Biochim Biophys Acta 2013; 1832: 57-66, doi: 10.1016/j.bbadis.2012. 10.003.

8. Huang J, Kodithuwakku ND, He W, Zhou Y, Fan W, Fang W, et al. The neuroprotective effect of a novel agent N2 on rat cerebral ischemia associated with the activation of PI3K/Akt signaling pathway. Neuropharmacology 2015; 95: 12-21, doi: 10.1016/j.neuropharm.2015.02.022.

9. Das G, Shravage BV, Baehrecke EH. Regulation and function of autophagy during cell survival and cell death. Cold Spring Harb Perspect Biol 2012; 4. pii: a008813, doi: 10.1101/cshperspect.a008813.

10. Nixon RA, Yang DS. Autophagy and neuronal cell death in neurological disorders. Cold Spring Harb Perspect Biol 2012; 4. pii: a008839, doi: 10.1101/cshperspect.a008839.

11. Son JH, Shim JH, Kim KH, Ha JY, Han JY. Neuronal autophagy and neurodegenerative diseases. Exp Mol Med 2012; 44: 89-98, doi: 10.3858/emm.2012.44.2.031.

12. $Y u$ Y, Feng L, Li J, Lan $X, A L$, Lv X, et al. The alteration of autophagy and apoptosis in the hippocampus of rats with natural aging-dependent cognitive deficits. Behav Brain Res 2017; 334: 155-162, doi: 10.1016/j.bbr.2017.07.003.

13. Perluigi M, Pupo G, Tramutola A, Cini C, Coccia R, Barone E, et al. Neuropathological role of PI3K/Akt/mTOR axis in 
Down syndrome brain. Biochim Biophys Acta 2014; 1842: 1144-1153, doi: 10.1016/j.bbadis.2014.04.007.

14. Uchiyama Y, Shibata M, Koike M, Yoshimura K, Sasaki M. Autophagy-physiology and pathophysiology. Histochem Cell Biol 2008; 129: 407-420, doi: 10.1007/s00418-0080406-y.

15. Xu J, Huai $Y$, Meng N, Dong Y, Liu Z, Qi Q, et al. L-3-nButylphthalide activates Akt/mTOR signaling, inhibits neuronal apoptosis and autophagy and improves cognitive impairment in mice with repeated cerebral ischemia-reperfusion injury. Neurochem Res 2017; 42: 2968-2981, doi: 10. 1007/s11064-017-2328-3.

16. Qi Q, Xu J, Lv P, Dong Y, Liu Z, Hu M, et al. DL-3-nbutylphthalide alleviates vascular cognitive impairment induced by chronic cerebral hypoperfusion by activating the Akt/Nrf2 signaling pathway in the hippocampus of rats. Neurosci Lett 2018; 672: 59-64, doi: 10.1016/j.neulet.2017. 11.051.

17. Wang G, Shi Y, Jiang X, Leak RK, Hu X, Wu Y, et al. HDAC inhibition prevents white matter injury by modulating microglia/macrophage polarization through the GSK3beta/PTEN/ Akt axis. Proc Natl Acad Sci USA 2015; 112: 2853-2858, doi: 10.1073/pnas.1501441112.

18. Luo $X Q$, Li $A$, Yang $X$, Xiao $X$, Hu R, Wang TW, et al. Paeoniflorin exerts neuroprotective effects by modulating the M1/M2 subset polarization of microglia/macrophages in the hippocampal CA1 region of vascular dementia rats via cannabinoid receptor 2. Chin Med 2018; 13: 14, doi: 10.1186/s13020-018-0173-1.

19. Li X, Hu X, Wang J, Xu W, Yi C, Ma R, et al. Inhibition of autophagy via activation of PI3K/Akt/mTOR pathway contributes to the protection of hesperidin against myocardial ischemia/reperfusion injury. Int $\mathrm{J} \mathrm{Mol}$ Med 2018; 42: 1917-1924.

20. Hang P, Zhao J, Su Z, Sun H, Chen T, Zhao L, et al. Choline Inhibits ischemia-reperfusion-induced cardiomyocyte autophagy in rat myocardium by activating Akt/mTOR signaling. Cell Physiol Biochem 2018; 45: 2136-2144, doi: 10.1159/ 000488049.

21. Song $Y$, Liu W, Ding $Y$, Jia $Y$, Zhao J, Wang $F$, et al. Salvianolic acid $\mathrm{A}$ ameliorates renal ischemia/reperfusion injury by activating Akt/mTOR/4EBP1 signaling pathway. Am J Physiol Renal Physiol 2018; 315: F254-F262, doi: 10. 1152/ajprenal.00508.2017.

22. Yan BC, Wang J, Rui Y, Cao J, Xu P, Jiang D, et al. Neuroprotective effects of gabapentin against cerebral ischemia reperfusion-induced neuronal autophagic injury via regulation of the PI3K/Akt/mTOR signaling pathways. J Neuropathol Exp Neurol 2019; 78: 157-171, doi: 10.1093/jnen/ nly119.

23. Qin $Y$, Hu W, Yang $Y$, Hu Z, Li W, Fang M. Neuroprotective effect of DAHP via antiapoptosis in cerebral ischemia. Behav Neurol 2018; 2018: 5050469, doi: 10.1155/2018/ 5050469.

24. Li W, Yang Y, Hu Z, Ling S, Fang M. Neuroprotective effects of DAHP and Triptolide in focal cerebral ischemia via apoptosis inhibition and PI3K/Akt/mTOR pathway activation. Front Neuroanat 2015; 9: 48.

25. Bottini G, Vallar G, Cappa S, Monza GC, Scarpini E, Baron P, et al. Oxiracetam in dementia: a double-blind, placebo-controlled study. Acta Neurol Scand 1992; 86: 237-241, doi: 10.1111/j.1600-0404.1992.tb05077.x.

26. Gschwind HP, Schutz H, Wigger N, Bentley P. Absorption and disposition of 14C-labelled oxiracetam in rat, dog and man. Eur J Drug Metab Pharmacokinet 1992; 17: 67-82, doi: 10.1007/BF03189990.

27. Ponzio F, Pozzi O, Banfi S, Dorigotti L. Brain entry and direct central pharmacological effects of the nootropic drug oxiracetam. Oxiracetam: brain entry and pharmacological effects. Pharmacopsychiatry 1989; 22: 111-115, doi: 10. 1055/s-2007-1014629.

28. Huang L, Shang E, Fan W, Li X, Li B, He S, et al. Soxiracetam protect against ischemic stroke via alleviating blood brain barrier dysfunction in rats. Eur J Pharm Sci 2017; 109: 40-47, doi: 10.1016/j.ejps.2017.07.029.

29. Malykh AG, Sadaie MR. Piracetam and piracetam-like drugs: from basic science to novel clinical applications to CNS disorders. Drugs 2010; 70: 287-312, doi: 10.2165/ 11319230-000000000-00000.

30. Kometani M, Okada M, Takemori E, Hasegawa Y, Nakao N, Inukai T. Effect of oxiracetam on cerebrovascular impairment in rats. Arzneimittelforschung 1991; 41: 684-689.

31. Baumel B, Eisner L, Karukin M, MacNamara R, Katz RJ, Deveaugh-Geiss J. Oxiracetam in the treatment of multiinfarct dementia. Prog Neuropsychopharmacol Biol Psychiatry 1989; 13: 673-682, doi: 10.1016/0278-5846(89)90054-7.

32. Yao XL, Yao ZH, Li L, Nie L, Zhang SF. Oxiracetam can improve cognitive impairment after chronic cerebral hypoperfusion in rats. Psychiatry Res 2016; 246: 284-292, doi: 10.1016/j.psychres.2016.10.006.

33. Gabryel B, Trzeciak HI, Pudelko A, Cieslik P. Influence of piracetam and oxiracetam on the content of high-energy phosphates and morphometry of astrocytes in vitro. Pol J Pharmacol 1999; 51: 485-495.

34. Fordyce DE, Clark VJ, Paylor R, Wehner JM. Enhancement of hippocampally-mediated learning and protein kinase $\mathrm{C}$ activity by oxiracetam in learning-impaired DBA/2 mice. Brain Res 1995; 672: 170-176, doi: 10.1016/0006-8993(94) 01389-Y.

35. Speetzen LJ, Endres M, Kunz A. Bilateral common carotid artery occlusion as an adequate preconditioning stimulus to induce early ischemic tolerance to focal cerebral ischemia. $J$ Vis Exp 2013; e4387.

36. Benito-Cuesta I, Diez H, Ordonez L, Wandosell F. Assessment of autophagy in neurons and brain tissue. Cells 2017; 6. pii: E25, doi: 10.3390/cells6030025.

37. Baskys A, Hou AC. Vascular dementia: pharmacological treatment approaches and perspectives. Clin Interv Aging 2007; 2: 327-335.

38. Jin X, Li T, Zhang L, Ma J, Yu L, Li C, et al. Environmental enrichment improves spatial learning and memory in vascular dementia rats with activation of Wnt/beta-catenin signal pathway. Med Sci Monit 2017; 23: 207-215, doi: 10. 12659/MSM.902728.

39. Brown WR, Thore CR. Review: cerebral microvascular pathology in ageing and neurodegeneration. Neuropathol Appl Neurobiol 2011; 37: 56-74, doi: 10.1111/j.1365-2990. 2010.01139.x.

40. Xu X, Li Z, Yang Z, Zhang T. Decrease of synaptic plasticity associated with alteration of information flow in a rat model 
of vascular dementia. Neuroscience 2012; 206: 136-143, doi: 10.1016/j.neuroscience.2011.12.050.

41. Cory S, Adams JM. The Bcl2 family: regulators of the cellular life-or-death switch. Nat Rev Cancer 2002; 2: 647656, doi: 10.1038/nrc883.

42. Fan $M$, Jin $W$, Zhao $H$, Xiao $Y$, Jia $Y$, Yin $Y$, et al. Lithium chloride administration prevents spatial learning and memory impairment in repeated cerebral ischemia-reperfusion mice by depressing apoptosis and increasing BDNF expression in hippocampus. Behav Brain Res 2015; 291: 399-406, doi: 10.1016/j.bbr.2015.05.047.
43. Rosello A, Warnes G, Meier UC. Cell death pathways and autophagy in the central nervous system and its involvement in neurodegeneration, immunity and central nervous system infection: to die or not to die--that is the question. Clin Exp Immunol 2012; 168: 52-57, doi: 10.1111/j.1365-2249.2011. 04544.x.

44. Chen L, Wei X, Hou Y, Liu X, Li S, Sun B, et al. Tetramethylpyrazine analogue CXC195 protects against cerebral ischemia/reperfusion-induced apoptosis through $\mathrm{PI3K} / \mathrm{Akt} / \mathrm{GSK} 3$ beta pathway in rats. Neurochem Int 2014; 66: 27-32, doi: 10.1016/j.neuint.2014.01.006. 